\title{
The effect of ear acupressure medicine at the "Shen Men" point on the nausea and vomiting during pregnancy
}

\author{
SANAZ NEHBANDANI ${ }^{1}$, HAJAR SALEHI ${ }^{2}$, KHADIJEREZAIE KEIKHAIE ${ }^{3 *}$, HOSSEIN RASHKI GHALENOW ${ }^{4}$, FATEMEH \\ MIRZAIE ${ }^{5}$, MAHIN BADAKHSH ${ }^{6}$ \\ ${ }^{1}$ Instructor, MSc of Midwifery, Faculty of Nursing and Midwifery, Zabol University of Medical Sciences, Zabol, Iran. \\ ${ }^{2}$ Student of Midwifery, Student Research Committee, Faculty of Nursing and Midwifery, Zabol University of Medical Sciences, Zabol, Iran. \\ ${ }^{3}$ Associated Professor of maternal fetal medicine, ZabolMedicinalPlants Research Center,Zabol University of Medical Sciences, Zabol, Iran. \\ ${ }^{4}$ MSc, Faculty of Nursing and Midwifery, Community Nursing Research Center, Zahedan University of Medical Sciences, Zahedan, Iran \\ ${ }^{5}$ MSc of Midwifery, Faculty of Nursing and Midwifery, Zabol University of Medical Sciences, Zabol, Iran. \\ ${ }^{6}$ MSc of Midwifery, Faculty of Nursing and Midwifery, Zabol University of Medical Sciences, Zabol \\ *Correspondence to Khadije Rezaie Keikhaie،E-mail:Rezaie.azar@ymail.com \\ ORCID:0000-0001-5389-7040
}

\begin{abstract}
Background: Nausea and vomiting during pregnancy is one of the most common gastrointestinal disorders that more than $85 \%$ of pregnant women experience. However, controlling and treating this complication is still one of the most important issues in antenatal care.

Aim: To investigate the effect of ear acupressure at Shen Men point on relieving nausea, vomiting and retchingduring pregnancy.

Methods: In this quasi-experimental study, 100 pregnant women with a gestational age of less than 16 weeks attending the health clinics ofZabol city during 2019-2020 were studied in two control and intervention groups ( $n=50$ in each group). The samples in the intervention group weretrained to apply pressure ontheirears'Shen Men point with the thumb for three minutes three times a day (morning, noon, and night), for a duration of one month. At the end of second and fourth weeks, the data were collected using the Rhodes index form and then, were analyzed by SPSS software version 22.

Results: According to the results, there was no significant difference between the two groups in terms of age, gestational age, occupation and education. The difference in the mean scores of nausea, vomiting and retching was not statistically significant between the control and intervention groups before the study. But four weeks after the study, a significant difference was observed in the mean scores of vomiting, nausea and retching between the two groups, so that the mean scores of vomiting, nausea and retching were significantly lower in the intervention group than in the control group.

Conclusion: The ear acupressure medicine at the Shen men point can be used as a non-invasive, safe and inexpensive method to relieve nausea, vomiting and retching during pregnancy.

Keywords :Acupressure medicine, Shen Men, Vomiting, Pregnancy, Nausea, Retching, Rhodosis
\end{abstract}

\section{INTRODUCTION}

Pregnancy is a normal condition that is accompanied by complaints and discomforts. Nausea and vomiting are two of the most common complaints of pregnancy, ranging from a mild reluctance to eat certain foods to severe vomiting ${ }^{1,2}$. Many women experience some digestive disorders during pregnancy, of which nausea and vomiting are the most common ones ${ }^{3,4}$. Nausea and vomiting during pregnancy occur in 50 to $90 \%$ of pregnancies. In $70 \%$ of cases, it starts between the $4^{\text {th }}$ and $6^{\text {th }}$ weeks of pregnancy and lasts until the $12^{\text {th }}$ to $14^{\text {th }}$ week and sometimes, even up to the $20^{\text {th }}$ week of pregnancy ${ }^{5}$. Despite their historical antiquity, their high prevalence and many researches that have been done in this area, nausea and vomiting during pregnancy and their exact causesare still unknown, so that hormonal, social, personal, psychological, racial and geographical factors are believed to be involved in their occurrence ${ }^{6}$. In recent years, various pharmacological and nonpharmacological treatments have been used to reduce the symptoms of gestational nausea and vomiting ${ }^{7,8}$, but since pregnant women refuse to take any medication during pregnancy, the only way to intervene in these people is to use non-pharmacological therapies. In modern medicine, the proposed solutions to control nausea and vomiting are the use of drugs, as they can control this problem by having a direct effect on the nervous system. The occurrence of unwanted side effects of chemical drugs is a problem that is not hidden from anyone. Nausea and vomiting, in addition to being highly annoying in early pregnancy, are one of the most common side effects of anesthesia and the patient needs effective measures to control these complications. On the other hand, the use of antiemetic drugs has unpleasant side effects, such as extrapyramidal syndrome, drowsiness and hypotension. These problems have led today's researchers to turn to other methods such as non-pharmacological therapies and complementary medicine $e^{1,5}$. Acupressure is one of the branches of acupuncture, with the difference that in this method there is no need to use a needle to stimulate the points. In fact, acupressure is a type of acupuncture, in which finger or hand pressure is used to stimulate the points that believed to cause different effects ${ }^{1,9,10}$. Studies have shown that acupressure improves nausea and vomiting in people. In the study of Saeedi et al (2016), which was performed on 92 pregnant women in Kerman, the combination of psychological counseling and acupressure training led to a reduction in the severity and duration of nausea and vomiting in the intervention group compared to the control group $\left.{ }^{6}\right)$. By investigating the effect 
of acupressure by C-Band on nausea and vomiting, Steele et al. (2000) reported a significant decrease in the frequency and severity of vomiting and nausea in the P6 acupressure group using C-Band ${ }^{11}$. In a study of Korean acupressure, Boehler et al. (2002) reported that this method significantly reduced nausea in women after laparoscopic surgery ${ }^{12}$.

A large number of pregnant women complain about nausea and vomiting during pregnancy and refer tothem as factorsthat disrupt their personal, family and social functions. They also worry about the detrimental effects of chemical drugs on the fetus during pregnancy ${ }^{6}$. So, considering the lack of study on the effect of acupressure at Shen Men point on gestational nausea and vomiting and also, taking into accountthe growing tendency to use acupressure in the treatment of many illnesses, such as nausea and vomiting during pregnancy all around the world, this study was conducted to evaluate the effect of acupressure at Shen Men point on the severity of nausea and vomiting during pregnancy.

\section{METHOD}

This is a quasi-experimental study that was conducted in 2019-2020on 100 pregnant women with a gestational age of $16-10$ weeks attending the health centers ofZabol city for the treatment of nausea and vomiting during pregnancy. Inclusion criteria in this study were;being literate, having a confirmed normal pregnancy based on the health center medical records, having nausea and vomiting during pregnancy, having singleton pregnancy, having gestational age of 10 to 16 weeks based on the ultrasound at the first trimester, absence of Molar or twins pregnancy and abortion threat symptoms, having no acute pyelonephritis, having no thyroid dysfunction and any unknown gastrointestinal disease, giving consent to participate in the study, and not taking any anti-nausea herbal or chemical medication during the last 24 hours prior tothe start of the study. Exclusion criteria included; unwillingness to continue with the study, participating in other educational programs at the same time, and having nausea / vomiting and threat of abortion. After approval of the research project by the Ethics Committee of Zabol University of Medical Sciences and obtaining permission from respected officials of the University, the researcher began tocollect the data. For this purpose, first the name of each health center was written on a small piece of paper and then,a person who was not part of the researcher was asked to pick one of the papers. The name written on the paper indicated the health center, in which the sampling was done. Thus, the researcher attended the selected health center and after introducingherself to the patients and informed them about the objectives and method of the study, reminded them that their information would be kept confidential and used only for research purposes. They were also asked to complete an informed consent form. Then, the Rhodes demographic questionnaire and Rhodes nausea and vomiting index were completed by the researcher through interviews with the participants. Those who met the inclusion criteria were divided into two groups of intervention and control by random allocation. Also, a code was assigned to each day of the week except for Friday. Then, at the beginning of each week, 3 codes were randomly assigned to the intervention group and 3 codes to the control group, and the researcher allocated the qualified individuals into the intervention or control groups on certain days based on the codes. An attempt was made to match all the subjects in the control group with the intervention group in terms of age and number of pregnancies. In the intervention group, the samples were asked to clean their outer earlobes with $75 \%$ alcohol. Then, the Shem men point (Figure 1) was determined and a white vegetable seed was attached to each point with a special non-latex adhesive.

Figure 1: The location of Shen Men point on the ear

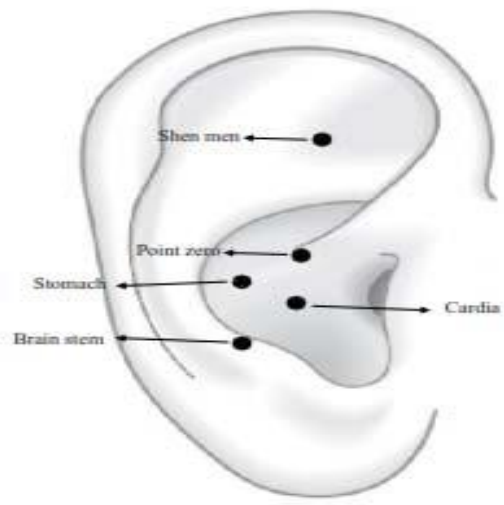

Patients were instructed to put a pressure on each point for at least three minutes three times a day (morning, noon, and night). Theseeds were placed on the earlobe for 5 days. The pressure technique was so that,a moderate stimulation would be done by pressing steadily and slightly increasing until felling a slight tingling or discomfort. Also, when the samples felt nauseous, they had to do it before vomiting. The researcher was in daily contact with the samples in order to follow up the trainingand answer their questions. In the control group, only routine care was performed and the patients did not receive any training. After two and four weeks, the samples were invited to health centers and they completed the questionnaires again.

Data collection tool was Rhodes nausea and vomiting index. The validity and reliability of thistool has been confirmed in previous studies ${ }^{13}$. The Rhodes nausea and vomiting index contains of 8 questions: Three questions are about nausea, including the frequency, duration, and degree of discomfort, each of which is scored between 0-4 withthe total score of $0-12$. Three questions are about vomiting including the frequency, amount, and degree of discomfort, each of which is scored between 0-4 with the total score of 0-12. Two questions are about retching including the frequency and amount of discomfort,each of which is scored between $0-4$ with the total score of $0-8$. The sum of scores for nausea, vomiting and retching is between 0-32.

In order to collect data, in addition to the demographic questionnaire, 7 forms of Rhodes nausea and vomiting index were given to the samples to enter information about nausea, vomiting and retching in the last 12 hours (three 
forms before and 4 forms after the intervention). To calculate the mean score of three days before the intervention, the score of each day was calculated separately and then the scores of all three days were added together and the final score was divided into three. Similarly, the score of 4 days after the intervention was calculated.

Data analysis: The collected data were recorded in Excel software version 2013. Then, they were entered into SPSS software version 22, and descriptive statistics (including frequency, mean and standard deviation) and inferential statistics (two-sample, independent and paired t-tests) were used to analyze the data.

\section{RESULTS}

In the present study, data analysis was performed on 100 pregnant women $(n=50$ in the control group and $n=50$ in the intervention group). Based on the findings of present study, there was nosignificant difference between the samples in the two groups in terms of demographic characteristics such as education level, occupation, age, gestational age and mass index $(p<0.05)$, which indicated their homogeneity (Table 1).

The results of two-sample and independent t-tests showed that before the study, there was no significant difference between the control and intervention groups in terms of nausea, vomiting and retching scores (Table 2, p
$<0.05)$. But two and four weeks after the intervention, a statistically significant difference was observed between the control and intervention groups in terms of nausea, vomiting and retching scores $(p<0.05)$. The mean scores of nausea, vomiting and retching in the intervention group that performed ear acupressure at Shen Men point were significantly lower than the control group $(p<0.05)$. The results of pairedt-test showed that the decrease in nausea, vomiting and retching scores was significant in the intervention group four weeks after the study compared to before study, while in the control group no significant difference was observed between the scores of nausea, vomiting and retching four weeks after the study compared to before study $(p<0.05)$.

The results of two-sample and independent t-tests showed that before the study there was no significant difference between the control and intervention groups in terms of total scores of nausea, vomiting and retching(Table $3, p<0.05$ ). But two and four weeks after the study, a statistically significant difference was observed between the control and intervention groups in terms of total scores of nausea, vomiting and retching ( $p<0.05)$. After the intervention, the total score of nausea, vomiting and retching in the control group $(7.94 \pm 1.61)$ was significantly lower than the intervention group (12.72 \pm 1.37).

Table 1: Demographic characteristics of pregnant women in the two groups of control and intervention

\begin{tabular}{|c|c|c|c|c|}
\hline \multicolumn{2}{|l|}{ Variable } & Control group $(n=50)$ & Intervention group $(n=50)$ & Significant level \\
\hline \multicolumn{2}{|l|}{ Age } & $28.2 \pm 3.06$ & $27.4 \pm 3.25$ & 0.124 \\
\hline \multicolumn{2}{|c|}{ Gestational age } & $9.60 \pm 2.14$ & $10.08 \pm 2.34$ & 0.287 \\
\hline \multicolumn{2}{|c|}{ Body mass index } & $23.56 \pm 1.83$ & $22.75 \pm 2.37$ & 0.057 \\
\hline \multicolumn{2}{|c|}{ Variable } & $\begin{array}{l}\text { Control group ( } n=50) \\
\text { Frequency (percentage) }\end{array}$ & $\begin{array}{llll}\begin{array}{l}\text { Intervention group } \\
\text { (percentage) }\end{array} & (n=50) & \text { Frequency } \\
\end{array}$ & Significant level \\
\hline \multirow{4}{*}{ Education } & Illiterate & $16(32)$ & $13(26)$ & \multirow{4}{*}{0.65} \\
\hline & Secondary & $15(30)$ & $21(42)$ & \\
\hline & Diploma & $11(22)$ & $10(20)$ & \\
\hline & University & $8(16)$ & $6(12)$ & \\
\hline \multirow[b]{3}{*}{ Occupation } & Housewife & $28(56)$ & $34(68)$ & \multirow{3}{*}{0.46} \\
\hline & Selfemployed & $16(32)$ & $12(24)$ & \\
\hline & Office worker & $6(12)$ & $4(8)$ & \\
\hline
\end{tabular}

Table 2: Comparison of the mean and standard deviation of the score of nausea, vomiting and retching before, during and after the intervention in the control and intervention groups

\begin{tabular}{|c|c|c|c|c|}
\hline \multicolumn{2}{|l|}{ Variable } & $\begin{array}{l}\text { Control group }(n=50), X \pm \\
\text { SD }\end{array}$ & Intervention group $(n=50), X \pm S D$ & Significant level \\
\hline \multirow{4}{*}{ Nausea } & Before the intervention & $5.68 \pm 1.02$ & $5.56 \pm 0.84$ & 0.52 \\
\hline & Two weeks after the intervention & $5.62 \pm 1.03$ & $5.16 \pm 1.13$ & 0.04 \\
\hline & Four weeks after the intervention & $5.54 \pm 0.95$ & $3.96 \pm 0.88$ & 0.000 \\
\hline & Significance level & 0.48 & 0.000 & \\
\hline \multirow{4}{*}{ Vomiting } & Before the intervention & $2.96 \pm 0.90$ & $2.92 \pm 0.85$ & 0.82 \\
\hline & Two weeks after the intervention & $3.02 \pm 0.32$ & $2.66 \pm 0.96$ & 0.01 \\
\hline & Four weeks after the intervention & $3.08 \pm 0.75$ & $2.02 \pm 0.65$ & 0.000 \\
\hline & Significance level & 0.53 & 0.000 & \\
\hline \multirow{4}{*}{ Retching } & Before the intervention & $3.84 \pm 0.91$ & $3.88 \pm 0.84$ & 0.82 \\
\hline & Two weeks after the intervention & $4 \pm 0.7$ & $2.56 \pm 0.70$ & 0.000 \\
\hline & Four weeks after the intervention & $4.10 \pm 0.76$ & $1.96 \pm 0.90$ & 0.000 \\
\hline & Significance level & 0.08 & 0.000 & \\
\hline
\end{tabular}

Table 3: Comparison of mean \pm standard deviation of total score of nausea, vomiting and retching in the two groups

\begin{tabular}{|l|l|l|l|}
\hline Nausea, vomiting and retching & Control group(n=50), X $\mathbf{S D}$ & Intervention group $(\mathbf{n}=\mathbf{5 0}), \mathbf{X} \pm$ SD & Significant level \\
\hline Before intervention & $12.48 \pm 1.49$ & $12.36 \pm 1.24$ & 0.66 \\
\hline Two weeks after the intervention & $12.64 \pm 1.29$ & $10.38 \pm 1.41$ & 0.000 \\
\hline Four weeks after the intervention & $12.72 \pm 1.37$ & $7.94 \pm 1.61$ & 0.000 \\
\hline Significance level & 0.41 & 0.000 & \\
\hline
\end{tabular}




\section{DISCUSSION}

Nausea and vomiting is one of the most common gastrointestinal disorders and one of the most important issues in pregnancy, which in addition to the physical complications, leads to psychological complications in pregnant women. Treatment of nausea and vomiting rarely leads to complete elimination of this probleminpregnant women, but this unpleasant feeling can be reduced with psychological support and lifestyle changes ${ }^{6,14}$, and also the use of various methods of alternative medicine. One of these methods is acupressure. Acupressure is a branch of acupuncture that is widely used in the treatment of nausea and vomiting in traditional Chinese medicine. The mechanism of action of acupressure in the treatment of nausea and vomiting is not clear. However, the possible mechanism, by which itaffectsthe nausea and vomiting, is to release beta-endorphins in the cerebrospinal fluid that affects hair receptors and increases gastric movements ${ }^{14}$. The aim of this study was to determine the effect of ear acupressure at Shen Men point (as a non-pharmacological intervention) on the nausea, vomiting and retchingof pregnant women. The results of this study showed that, the ear acupressure at Shan Men point was effective in reducing nausea, vomiting and retching ofpregnant women inthe first trimester of pregnancy. According to the results, before the study, no significant difference was observed between the two groups in terms of nausea, vomiting and vomiting scores. However, two and four weeks after the intervention, a decrease in the scores of nausea, vomiting and retching was observedin theintervention group compared to the control group. Decreased scores of nausea, vomiting, and retching under the influence of acupressure have been reported in several studies ${ }^{15,16}$. In the study of Chen et al (2005), a statistically significant difference in the score of nausea, vomiting and retching was observed in the intervention group ${ }^{17}$, which is consistent with the results of present study. Eghbali et al. (2015) reported that, the use of acupressure on the ear, heart, stomach, and central nervous system points along with anti-nausea and vomiting medications can lead to a reduction in acute nausea and vomiting ${ }^{18}$, which is in line with the findings of present study. In a study, Yeh et al (2012) reached similar results and reported that the use of ear acupressureat sham auricular points in children with acute lymphoblastic leukemia led to a significant reduction in the frequency and severity of nausea at acute and delayed phases ${ }^{19}$. A research showed that the lowest score of nursing students is in drug calculation related to the calculation of regulation of droplets of solution and infused drugs $^{20}$. Meanwhile, Puangsricharern and Mahasukhon (2008) showed that, although ear acupressure at the origin point reducedthe nausea and vomiting in early pregnancy, this reduction was not statistically significant, so they concluded that, the application of ear acupressure at the origin pointcannot reduce the frequency and severity of nausea and vomiting during pregnancy ${ }^{21}$. Thisresult is not consistent with the findings of present study. This difference in the results of this study and present research may be due to the use of different ear acupressure pints in these two studies. person's knowledge of an issue and his or her insight and performance, it is hoped to raise the awareness andimprove individuals' performance through such methods as conducting health and medical education sessions $^{22}$.

\section{CONCLUSION}

Based on the results of present study, it can be concluded that the use of ear acupressure at the Shen Men point can be effective in reducing nausea and vomiting during pregnancy. Therefore, by providing appropriate facilities and educational programs on how to perform acupressure, midwives and nurses can apply this complementary treatment method along with other medical procedures to reduce the vomiting, nausea and retching in pregnant women.

Ethics approval: Prior to the study, ethical approval (IRZBMUREC1398096) was obtained from the faculty of Medical Sciences at Zabol University. This study adhered to the principles of the Declaration of Helsinki. The ethical principles of autonomy, confidentiality, and anonymity were considered for the participants. In order to enter the study, all participants were asked to provide an oral and written informed consent, and their participation in the study was optional. Before taking part in the study, the participants were informed about the purpose and method of the study, and a written informed consent was collected. All ethical measures including honesty in the provision of results, data confidentiality, and anonymity were considered with care.

Ethics approval and consent to participate: This study was approved by the ethics committee at Zabol University of Medical Sciences (NO 1398096 DATE 2019/10/13). Autonomy, independence, confidentiality and privacy of the participants were considered. Participants were informed about the study. We obtain written and oral informed consent for interview and take notes. The participants were free to exit the study whenever.

Consent for publication: All participant was assured of their confidentiality and privacy for data analysis and reporting.

Availability of data and materials: The datasets using in the study are available from the corresponding author on reasonable request.

Competing interests: The author(s) declared no potential conflicts of interest with respect to the research, authorship, and/or publication of this article.

Funding: 'Not applicable'

Authors' contributions: All authors have read and approved the manuscript. All authors contributed to conceptualization, methodology, writing- original draft preparation, and writing- reviewing and editing.

Acknowledgement: The present article ispart ofa research project atZabol University of Medical Sciences, which was registered in the University's Ethics Committee with the number: IRZBMUREC1398096. The authors would like to thank all those who helped us in this study.

\section{REFERECES}

1. Ozgoli G SS, Rassaian N, Alave majd H. Effect of acupressure with wristband on nausea and vomiting of pregnancy. 2009;7(3). 
2. Tara F, Bahrami-Taghanaki H, Ghalandarabad MA, ZandKargar Z, Azizi H, Esmaily H, et al. The effect of acupressure on the severity of nausea, vomiting, and retching in pregnant women: a randomized controlled trial. 2020:1-8.

3. Tadayon M, Salehian T, Abbaspour z, Latifi SM. Comparison of the effect of acupressure and vitamin B6 on the severity of nausea and vomiting in pregnancy. Jundishapur Scientific Medical Journal.

4. Gürkan ÖC, Arslan HJCTiCP. Effect of acupressure on nausea and vomiting during pregnancy. 2008;14(1):46-52.

5. Seidaghamiri Z.B. HNS, Ramezanzadeh F., Haghollahi F., Vijeh M. Effect of acupressure by Sea-Bands on nausea and vomiting of pregnancy. Payesh. 2008;7(4):369-74.

6. Zahra Saeedi KA, Firoozeh Mirzaee, Atefeh Ahmadi, Tania Dahesh. The effect of combination of psychological counseling and acupressure training with couple therapy approach on nausea and vomiting in pregnancy. The Iranian Journal of Obstetrics, Gynecology and Infertility. 2019;21(11):71-9.

7. Einarson A, Maltepe C, Boskovic R, Koren GJCfp. Treatment of nausea and vomiting in pregnancy: an updated algorithm. 2007;53(12):2109-11.

8. Jin JJJ. Treatments for Nausea and Vomiting During Pregnancy. 2016;316(13):1420-

9. Steele NM, French J, Gatherer-Boyles J, Newman S, Leclaire S. Effect of Acupressure by Sea-Bands on Nausea and Vomiting of Pregnancy. Journal of Obstetric, Gynecologic, Neonatal Nursing. 2001;30(1):61-70.

10. Salehian $T$, Delaram $M$, Tadayon $M$. The effect of acupressure using $\mathrm{C}$-band on the severity of pregnancy nausea and vomiting. MEDICAL JOURNAL OF HORMOZGAN UNIVERSITY.11(1):77-82.

11. Steele NM, French J, Gatherer-Boyles J, Newman S, Leclaire SJJoO, Gynecologic,, Nursing N. Effect of Acupressure by Sea-Bands on Nausea and Vomiting of Pregnancy. 2001;30(1):61-70.

12. Boehler M, Mitterschiffthaler G, Schlager A. Korean hand acupressure reduces postoperative nausea and vomiting after gynecological laparoscopic surgery. Anesthesia Analgesia. 2002;94(4):872-5.

13. Sh N, Gh SA, Kordi M. Efficacy of acupressure on reduse nasea and vomiting in the first trimester of pregnancy. Med $\mathrm{J}$ Mashhad Univ Med Sci. 2000;24(66):89-95.

14. Sima khavandi zadeh Aghadam BM. Evaluation of the Effects of Acupressure by Sea Band on Nausea and Vomiting of Pregnancy. The Iranian Journal of Obstetrics, Gynecology and Infertility. 2010.

15. Boehler M, Mitterschiffthaler G, Schlager AJA, Analgesia. Korean hand acupressure reduces postoperative nausea and vomiting after gynecological laparoscopic surgery. 2002;94(4):872-5.

16. Schlager A, Boehler M, Pühringer FJBJoA. Korean hand acupressure reduces postoperative vomiting in children after strabismus surgery. 2000;85(2):267-70.

17. Chen H-M, Chang F-Y, Hsu C-T. Effect of acupressure on nausea, vomiting, anxiety and pain among post-cesarean section women in Taiwan. The Kaohsiung journal of medical sciences. 2005;21(8):341-50.

18. Eghbali M, Varaei S, Jalalinia SF, Aalam Samimi M, Sa'atchi $\mathrm{K}$, Yekaninejad MS. Effect of auricular acupressure on acute nausea and vomiting induced by chemotherapy among breast cancer patients. Journal of hayat. 2015;21(2):29-39

19. Yeh CH, Chien L-C, Chiang YC, Lin SW, Huang CK, Ren D. Reduction in nausea and vomiting in children undergoing cancer chemotherapy by either appropriate or sham auricular acupuncture points with standard care. The Journal of Alternative Complementary Medicine. 2012;18(4):334-40.

20. Robabi H., Mahmoudi N., Arbabisarjou A., Movahed S. Drug calculation skills in nursing students of Zahedan University of medical sciences, Drug Invention Today. 2019,12(2):227-230

21. Puangsricharern A, Mahasukhon S. Effectiveness of auricular acupressure in the treatment of nausea and vomiting in early pregnancy. Med Assoc Thai. 2008;91(11):1633-8.

22. Robabi H., Ostad Kalayeh Mogharabi Sh.,Irandegani F. Arbabisarjou A., Evaluation of the performance and knowledge of youth and middle-aged population of Zahedan about diabetes mellitus, 2019,12(12): 2888-2891. 